\title{
Implementation of Smart-Learning Content Authoring Tool System Utilizing HTML5
}

\author{
Hyun-Seo Hwang $^{1 *}$, Jun-Soo Yun ${ }^{1}$, Jin-Tae Park ${ }^{1}$ and Il-Young Moon ${ }^{1}$ \\ ${ }^{1}$ Department of Computer Science and Engineering, Korea University of \\ Technology and Education, Cheon-an 330-708, Korea \\ [E-mail: smilebear1@koreatech.ac.kr]
}

\begin{abstract}
E-Learning industry, since 2008, while record high suit rate of 9.5 percent annually, was rapidly emerging in high value-added service industry of the next generation 1-2]. As information technology develops, several types of e-learning have changed. I recent years, smart learning using mobile devices has become a trend [3-4]. However, problems occur when the contents, solutions, or services implemented on existing PC-based systems are applied to a mobile environment [5]. Existing edearning mainly differs as was desktop PC-based operations, the internet connection as well, such as location-based services and augmented reality, can be applied a variety of techniques, it takes advantage of smart devices, and then, the existing e-learning became to provide differentiated services [6-7]. Most domestic technologies are developed ysing Flash and ActiveX, depending on the vendor. Cloud storage sistem that cloud computing providers provides large amounts of data storage and processing of cloud computing is a key component [10]. Most of the e-learning systems that use these some technologies are problematic to use in a smart-learning environnent [8]. To solve these problems, we implemented a user-oriented contents authoring tool which is appropriate for smart-learning [9]. By providing this mobile device-based authoring tool, users are able to create real-time content. Furthermore, we aim to use HTML5 technology to create a cross-platform tool and to improve the user-oriented environment and e-learning services available for smart phones. Our tool provides additional convenience to many users by building a mobile device, web, and user-log-server. Using this approach, the real "smart" form of the contents us din smart phones expected.
\end{abstract}

Keywords: HTML5, Mobile Device, MOOC, Smart Learning, Smartphone

\section{Introduction}

Education and information technology are the new growth engine of the e-learning industry allowing e-learning to become the world's fastest growing market in the knowledge services industry. E-learning means learning via electronics and information ommunication is performed by utilizing radio and broadcast technology. E-Learning mdustry, since 2008, while record high suit rate of 9.5 percent annually, was rapidly emerging in high value-added service industry of the next generation. The e-learning environment is extended from its PC-based origins to smartphones, making it possible to use without time and place constraints. In the world market, smart learning has spread rapidly to new technologies such as smart devices, allowing e-learning to integrate with smart phones and tablet PCs. Smart learning is a kind of education that utilizes electronic equipment, and is a concept also make defined concept that is included in u-learning. Existing e-learning mainly differs as was desktop PC-based operations, the internet connection as well, such as location-based services and augmented reality, can be applied a variety of techniques, it takes advantage of smart devices, and then, the existing e- 
learning became to provide differentiated services. According to the National IT Industry Promotion Agency, the domestic smart-learning market size is expected to grow to greater than three trillion this year. Around the world, this market has expanded the smart school business infrastructure built with government-led, smart school related market rapid growth is expected. However, there is also a problem that occurs in the smart learning services provided. There is a discomfort that learners receive provide a lecture for compatibility issues of mobile equipment. To solve those problems, this study implemented the user-oriented contents authoring tool system which is proper for the smart-learning. By providing this mobile device-based authoring tool, users will be able to create real-time contents. Furthermore, the aim is to use HTML technology but not to depend on OS, to support cross platform, and to build the user-oriented environment and e-learning service in smart phones. It also provides convenience to many users by building a mobile device, web, and user-log-server. These studies are expected to contribute to the development of efficient educational method that meets the future of smart running era.

\section{Related Research}

\subsection{Smart Learning}

Smart learning is the learning content and solu ions that use smart phones and mobile devices such as the tablet PC. Smart learning is simply not mean to be e-learning in another form using the mobile device or the smart devices Citing benefits of smart learning is learning seep into the life ouf of the formal form can quickly obtain the required information or knowledge. Smartphone, while the prevalence of mobile devices such as tablet PC popularity mobility, personalization, mobile devices are having such a characteristic can learn timeliness of the content that can be implemented by using the most appropriate device.

\subsection{Massively Open Online Course}

Recently, online public lectures that globally Massive Open Online Course (MOOC) has attracted attention. MOOC is a arge-scale online public lecture through which you can receive the eatcation of content through anyone without restrictions online. Lectures provided by the MOOC include course information, courses, quizzes, essays, and discussion, are constituted by a question bulletin board. In addition, the MOOC is enclosing three important meanings. First, humans deserve a good education; second, these innovative solutions reduce training costs. The third is the place of formation of the intellectual network. As an indicator of the new era of lifelong education, a number of universities are opening a MOOC web site by themselves, seeking alternatives of original online education beyond national borders. It is expected how the MOOC will change the education marketplace in the future.

\section{System Implementation}

Figure 1 describes the system structure chart. To realize the given system, it has been divided into three parts: Smart Learning Management web, Content Authoring Application, and Cloud Server. The Smart Learning Management web is for managing images users take or music users record through a web site based on PC environment, and the output (image or music) is printed as a Learning object form. Then the printed output is uploaded to the cloud server through a cloud interface. Moreover, the cloud interface, the user is able to confirm the uploaded files, other Learning Object, can upload the video and photo files. Through the Content Authoring Application, users produce and edit contents by themselves. Afterward the authored contents by the users are uploaded to the 
cloud server to be managed by the Smart Learning Management web. Figure 2 illustrates a user scenarios.

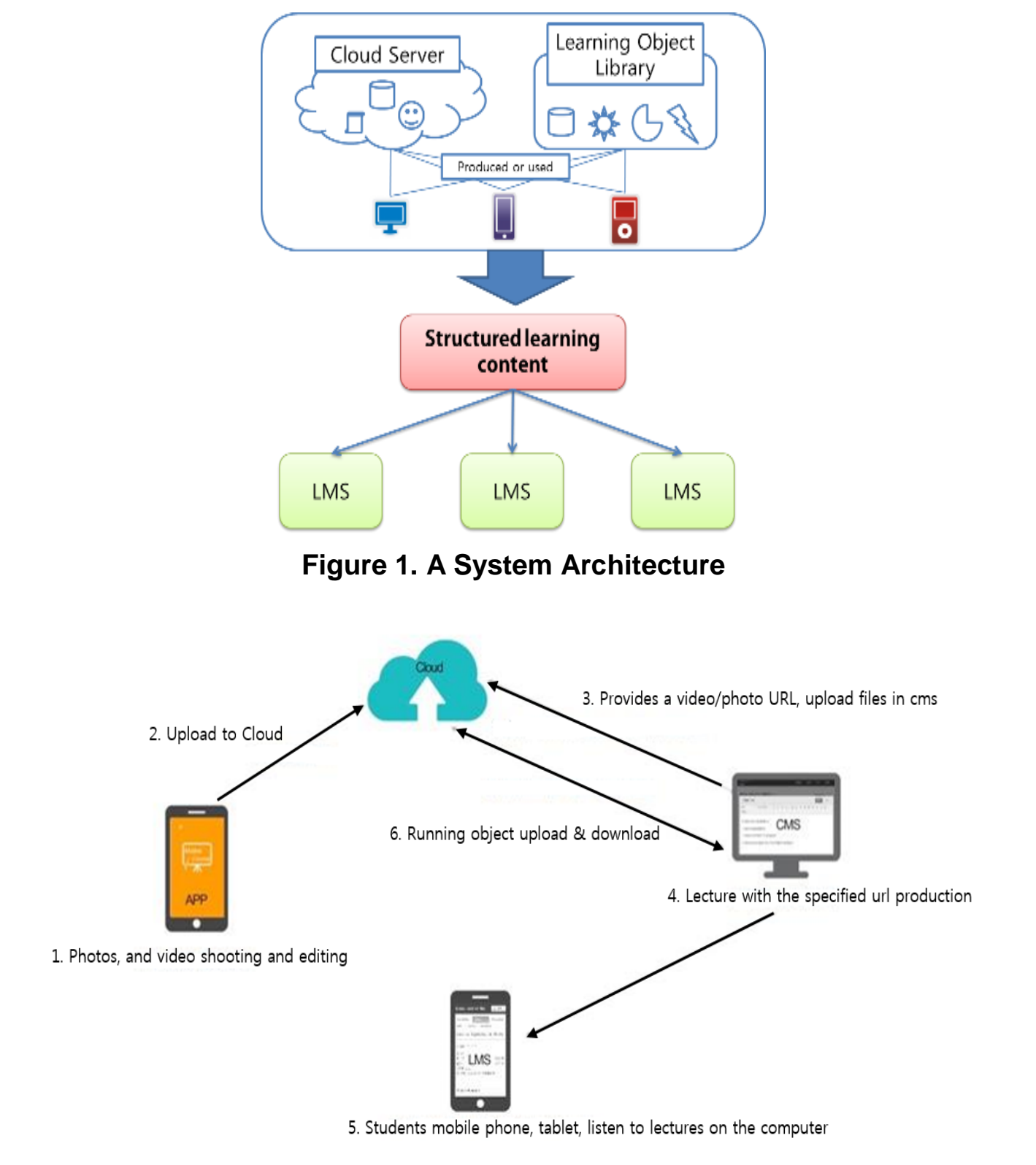

Figure 2. User Scenarios

\subsection{Smart Learning}

smart Learning Management web, designed based on the edx-platform, allow users to manage the contents by themselves. This web site has a number of functions such as controlling video/audio, linking transaction, publishing and viewing contents. The convenience is even more enhanced as it has been produced based on responsive-web site system, which let it work on both PC version and Mobile/Tablet version. Figure 3 describes the Smart Learning Management Web. Smart Learning Management web is edx - has been developed based on the platform. The LMS and CMS After creating the basic Web site based on the resolution 760px, when the size of the browser is smaller than the reference size, by changing the layout, mainly place the description and index river next, contents are down to below, each component, you configure the layout to use the entire 
screen. In addition, to login to put the account information to the cloud interface with post format when you log in to the CMS in order to link the CMS and the cloud. Together was allowed to $\log$ in and to keep the cloud session, so as to use when calling the cloud url. Cloud url page to configure the UI of the upload page of already had a file as it is so that there is no sense of incongruity to use. In the cloud url page, split only the name of the video url and video and read the source of the cloud of interface in ajax, to configure the table as a result of value. When you upload from the CMS to the cloud, it is sent directly upload the data to the cloud of interface. In the CMS after the data has risen to the cloud, re-read the updated file I so as to display the added screen page. The user, after selecting the video component in the unit of making a lecture by copying the individual url, paste the address. Since, it was configured such that lecture that has been created in the user look. Execution object is downloaded to the pc in tar format when you export the lecture in order to upload the running object to the cloud. This cloud is directly uploaded to, and is stored in the cloud of the running object folder. Thereafter, by utilizing the lecture import function files, it is designed to load the saved course. Running object, folder/as shown in Figure 4 starts from is composed course.xml. About, there is guidance of lecture, static, the video was used in the lecture, there is a material such as an mage, as policies that the students scored on a lecture takes place, video, vertical, sequential, html, video contents of the lecture but have you have the reference value of sub data.

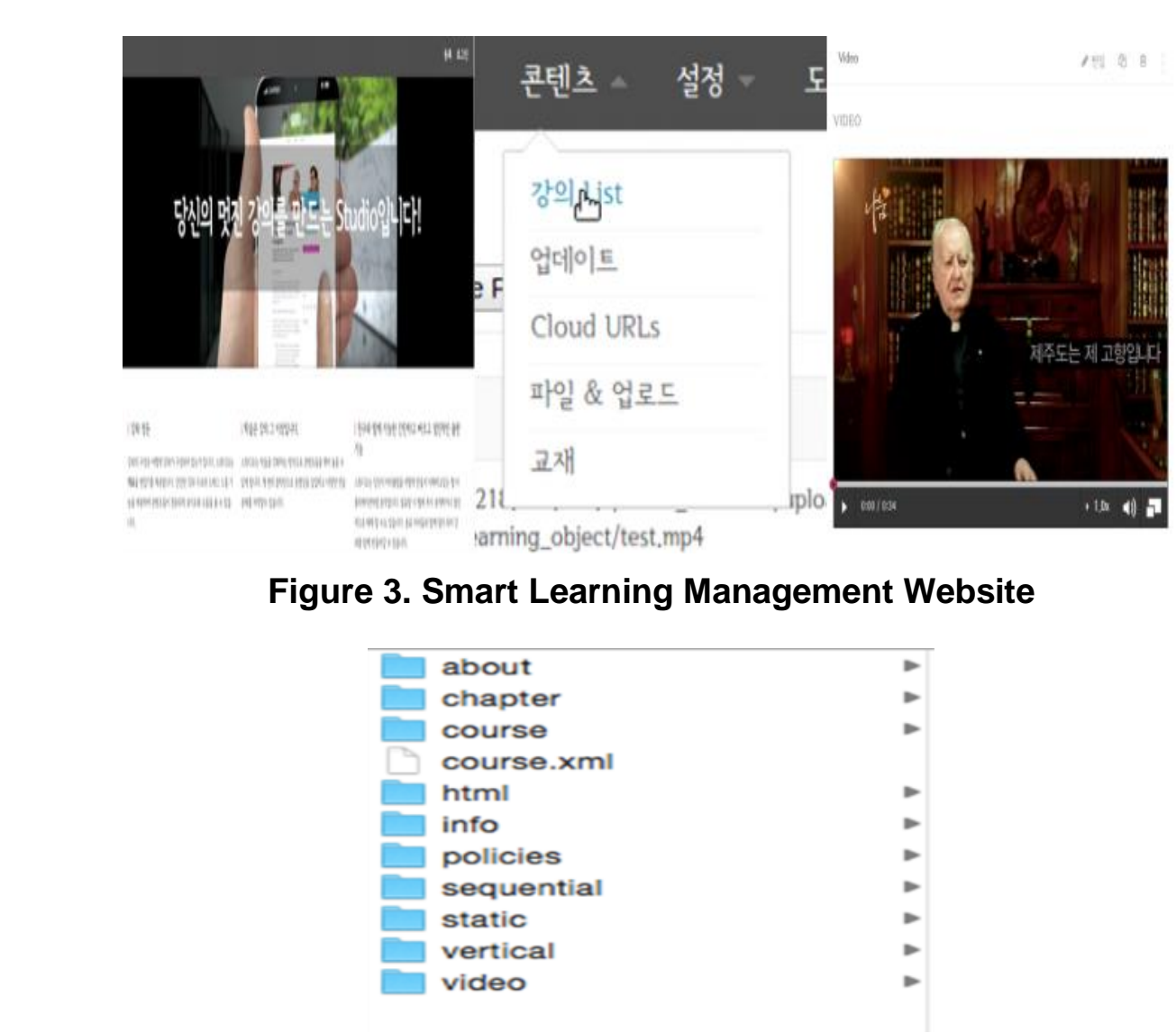

Figure 4. Running Object Structure

\subsection{Content Authoring Application}

Users can author their own lecture contents via the Content Authoring Application on their smart phone. This application consists of 'My lecture', which allows users see their lectures, 'Make lecture', where users can produce their own lecture contents, 'Lecture 
edit', which lets users add subtitles, give filtering effects, and edit the images, and 'Lecture upload', where the completed images can be uploaded. Figure 5 shows the Content Authoring Application. The user must be able to determine the content of which he is making. Therefore, to create a menu that my lecture, the menu was to check the photos and videos that are stored on your smartphone using the gallery features of the device. The media player can use the default media player built into the smartphone. The default media player is used to improve the compatibility of smartphones for multiple users. The user can easily configure the 'lecture manufacture' menu to create a teaching content. The user can take a picture and the video using the camera, which is built-in the smart phone, the captured image is stored in the smart phone. Videos are saved to the location specified by the make_course smartphone sdcard, not to be confused with existing video files. Uploading completed before the content in the cloud server, the user can edit the lecture in the desired direction. 'Edit lecture' so as to generate a first project. It can be edited and uploaded to the project units. Inside the project, it can be prepared by the method of combining the single image by adding the number of images stored in the smart phone. When switched to the next image in the current image it can beraded to a transition effect designated in advance. In addition, the user can create a video of the atmosphere they want to insert the background music. And a gradient, sepia and the like can be applied to the image filtering effect. And the subtitle function is needed for content production. In content creation applications, users gan put in the middle lower part of the video captions at any time. The edited video is stored in the interior of the user smart phone through the encoding process. The video is saved in the specified folder location. Made through the 'Edit lecture' content is rploaded to the cloud server through the 'Upload lecture'. Content is uploaded to the specified php file using httpPost, httpClient way. Before uploading your content to-implement adogin function on the basis of the information stored in the cloud server, cloud interface. When post content in the application, 'user email' folder is created on the cloud server. Three folders ('video', 'image', 'learning_object') are created inside the created folder. Each folder is stored by classifying the content uploaded by the user. In addition, upload.php received upload request to post a Request Ior Information to save your e-mail, file names, file paths, and saved in the specified database.

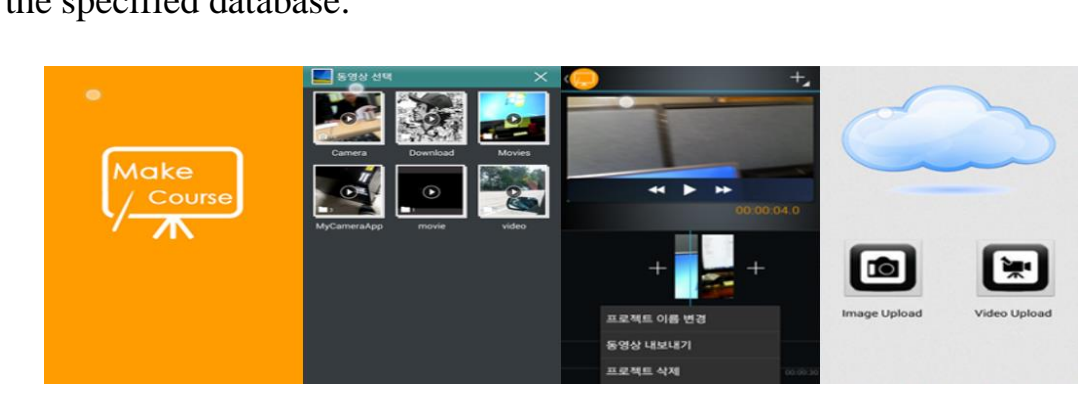

Figure 5. Content Authoring Application

\subsection{Building a Cloud Service}

A total of 3 nodes are required to construct the Cloud server. The Controller node is composed of 'MySQL' for database, 'RabbitMQ' for message service, 'Keysone' for service certification, ;Glance' as an image service, 'Nova' as a computer service and 'Horizon' as a dashboard service. We used Nova network to construct the system, not installing any other extra network service. To compute nodes, hypervisor KVM is installed, nova-network is installed together, and supports network service instances. The controller node, interface or requires the compute nodes for monitoring and management interface for connection with the controller node, constructed to have an interface or so two interfaces for communication with an external network. 


\subsection{Cloud Interface}

In the proposed model in this paper, in the cloud, save and manage the resources received from the application, its resource LOAD in Edx to create one of the completed course, again a course that has been created in the run object format, it will be uploaded or downloaded to the cloud. All work is facilitated, for user convenience, it is used in building a cloud interface separately. Cloud interfaces, it is possible to confirm the unique resource for each user, it is possible to manage the courses that have been created. Have been prepared on the basis of HTML and JS, as well as PC, it was also developed in the available reaction hybrid web in a mobile environment. Figure 6 shows a cloud interface.

\section{Conclusion}

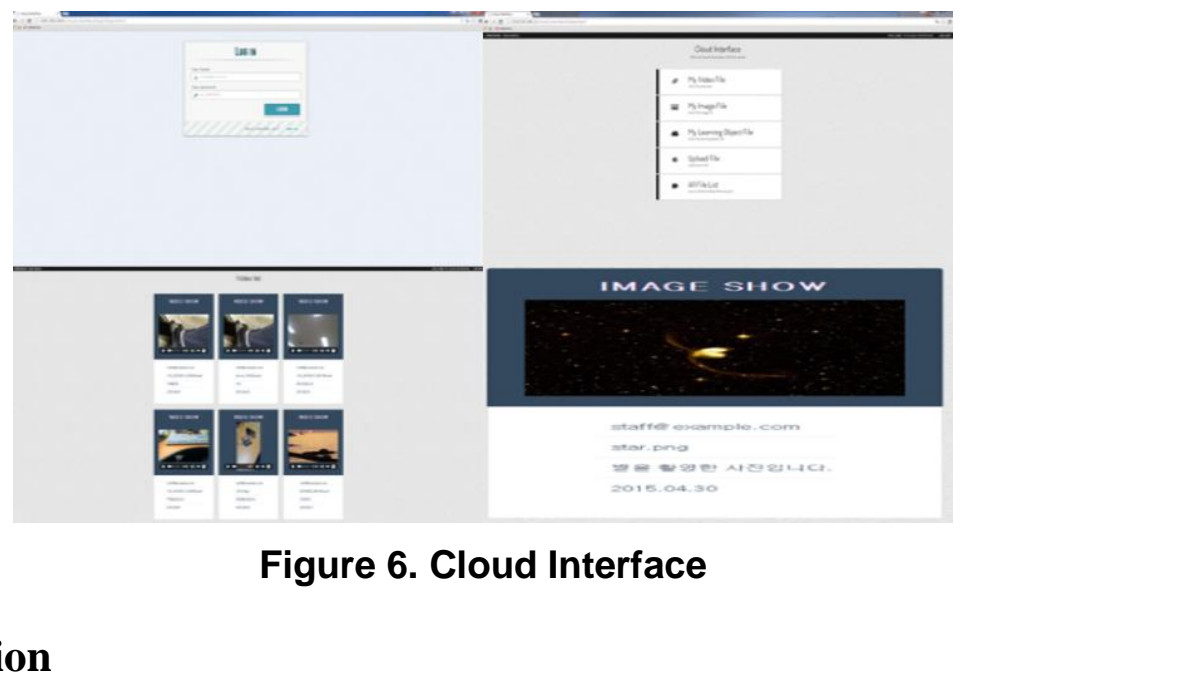

E-learning industry is well lnown around the world, and it is becoming more important. E-learning is defined the use of electronic educational technology in teaching and learning, it features lower environment impact as the PC and smart phone environment is getting larger. Recently, worldwide online open class that MOOC has attracted attention. MOOC anyone without restriction is a large online public courses can be educated in better through line. Lecture course information provided by the MOOC, and is configured courses, quizzes, essays, discussions, such as in the question bulletin board. In addition, MOOC have included the three important meaning. First, it is intended to be taken inte account the right to be able to receive a good education in one of the human, the second is that it innovative solutions for reducing training costs. And the third, it's the fact that they prace the formation of the intellectual network. Anyone as online courses to provide a system that was implemented in this paper also MOOC, has developed to be able to provide online courses via the Internet from anywhere. However, many experts have pointed out the limitations of smart running and MOOC. It is a smart running that combines information technology and e -learning, but it and the MOOC the user o lower the educational effects of inconvenience in the students to use is that there is a difficulty in the domestic introduction for the barriers of language. Nevertheless, there are some problems about portability when it need to connect with mobile devices. To figure out this problem, implementing user-oriented contents authoring tool system is required. This system will include Smart Learning Management web, Content Authoring Application and Cloud Server. Smart Learning Management web is needed to be developed based on Edx-platform, and it can help for users to author their own contents, to edit the contents and to upload them to cloud server by using the Content Authoring Application. Following this, the Cloud server can manage the files which were sent systematically. These studies are expected to contribute to the development of efficient educational method that meets the future of smart running era. 


\section{References}

[1] J. E. Moon, B. J. Kim, Y. H. Park, H. R. Im, Y. J. Hong, C. J. Ban, and C. J. Park, "Web and app development for student's assessment management in smart learning environment," The Korea Contents Association Conference, (2014), pp. 87-88.

[2] H. H. Kim and S. H. Shim, "Application method of web-based contents for smart learning environments," Korean Society for Internet Information, vol. 12, no. 2, (2011), pp. 235-236.

[3] J. H. Lee, J. H. Song, J. M. Oh, Y. J. Choi, and N. M. Moon, "The study on improvement of moving picture content authoring tool for usability enhancements", Journal of the HCI Society of Korea, vol. 10, no. 1, (2010), pp. 242-245.

[4] J. B. Kim, "Study on construction method of hybrid web-based smart learning," Journal of the Institute of Electronics and Information Engineers, vol. 49, no. 9, (2012), pp. 370-378.

[5] J. T. Park, J. S. Yun, O. Y. Kwon, I. Y. Moon, and B. J. Kim, "Development of a smart-learning platform and content authoring tool that uses a cloud and web," International Journal of Applied Engineering Research, vol. 9, no. 24, (2014), pp. 29869-29882.

[6] S. Y. Kim and J. A. Park, "Designing a Framework of Multimodal Contents Creation and Playback System for Immersive Textbook," Journal of the Korea Contents Society, vol. 10, no. 8, (2010) pp. 14 10.

[7] J. G. Lee, J. W. Lee, "Interface for in-situ Authoring of Augmented Reality Contents on a Mobile Device Environment," Journal of the Korea Contents Society, vol. 10, no. 7, (2010), pp 1-7

[8] J. H. Ra, S. H. Han, B. Y. Sung and Y. K. Kim, "Open Source Cloud Platforms: OpenStack and Cloud Stack", Communications of the Korean Institute of Information Seientists and Engineer, vol. 39, no. 1, (2012), pp. 259-261.

[9] J. H. Lee, J. H. Song, J. M. Oh, Y. J. Choi and N. M. Moon. "The study on Improvement of Moving picture content authoring tool for usability enhancements", Joumal of the HCI Society of Korea, vol. 2010, no. 1, (2010), pp. 242-245.

[10] J. S. Park, U. M. Bae and S. J. Jung, "Technical analysis of Cloud Storage for Cloud Computing," Journal of Information and Communication Convergence Engineering -col. 19, no.5, pp. 1129-1137.

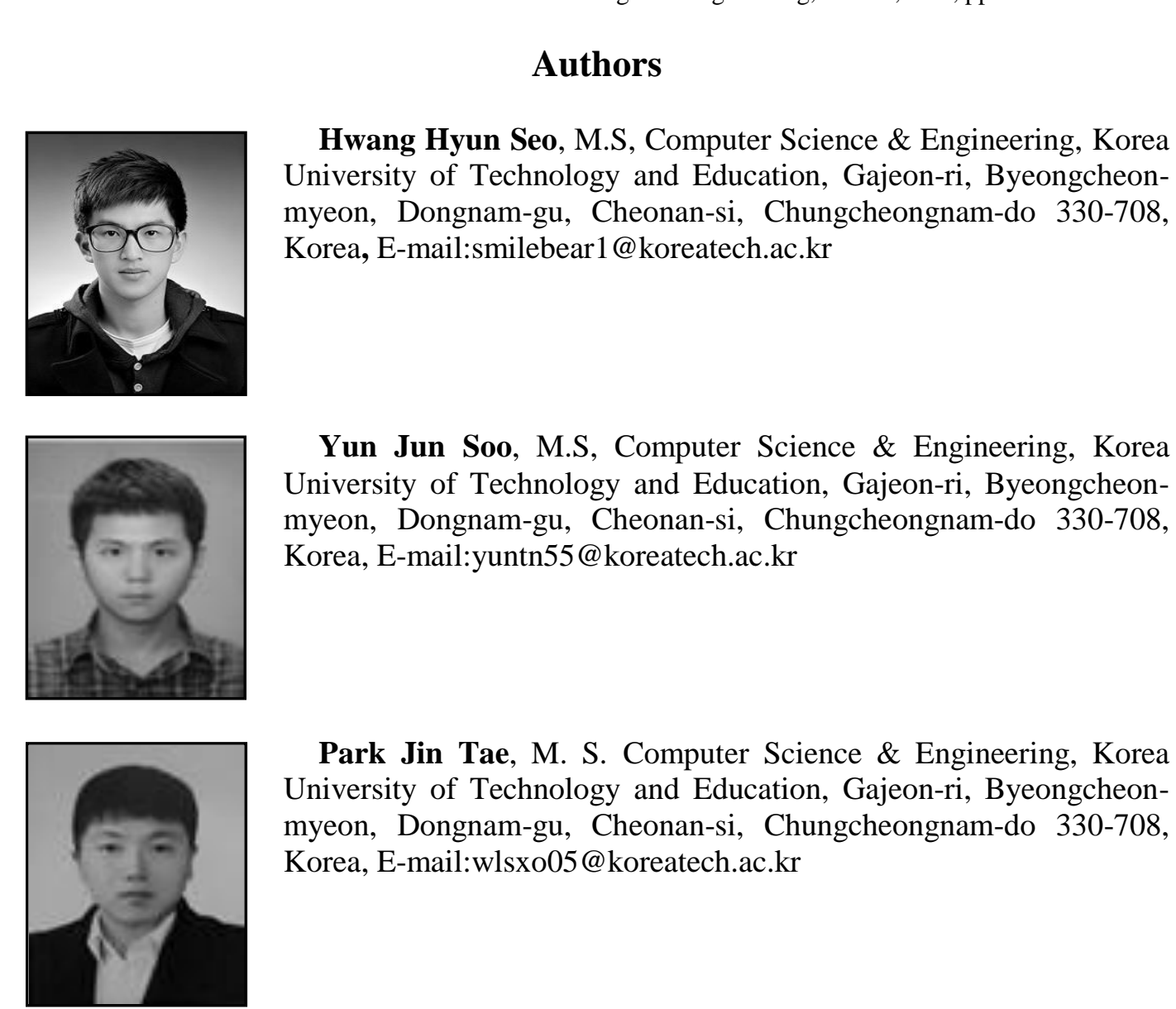




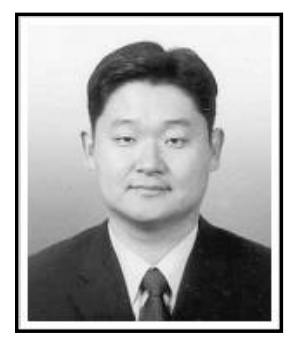

Moon Il Young, Ph. D. Computer Science \& Engineering, Korea University of Technology and Education, Gajeon-ri, Byeongcheonmyeon, Dongnam-gu, Cheonan-si, Chungcheongnam-do 330-708, Korea, E-mail:iymoon@koreatech.ac.kr

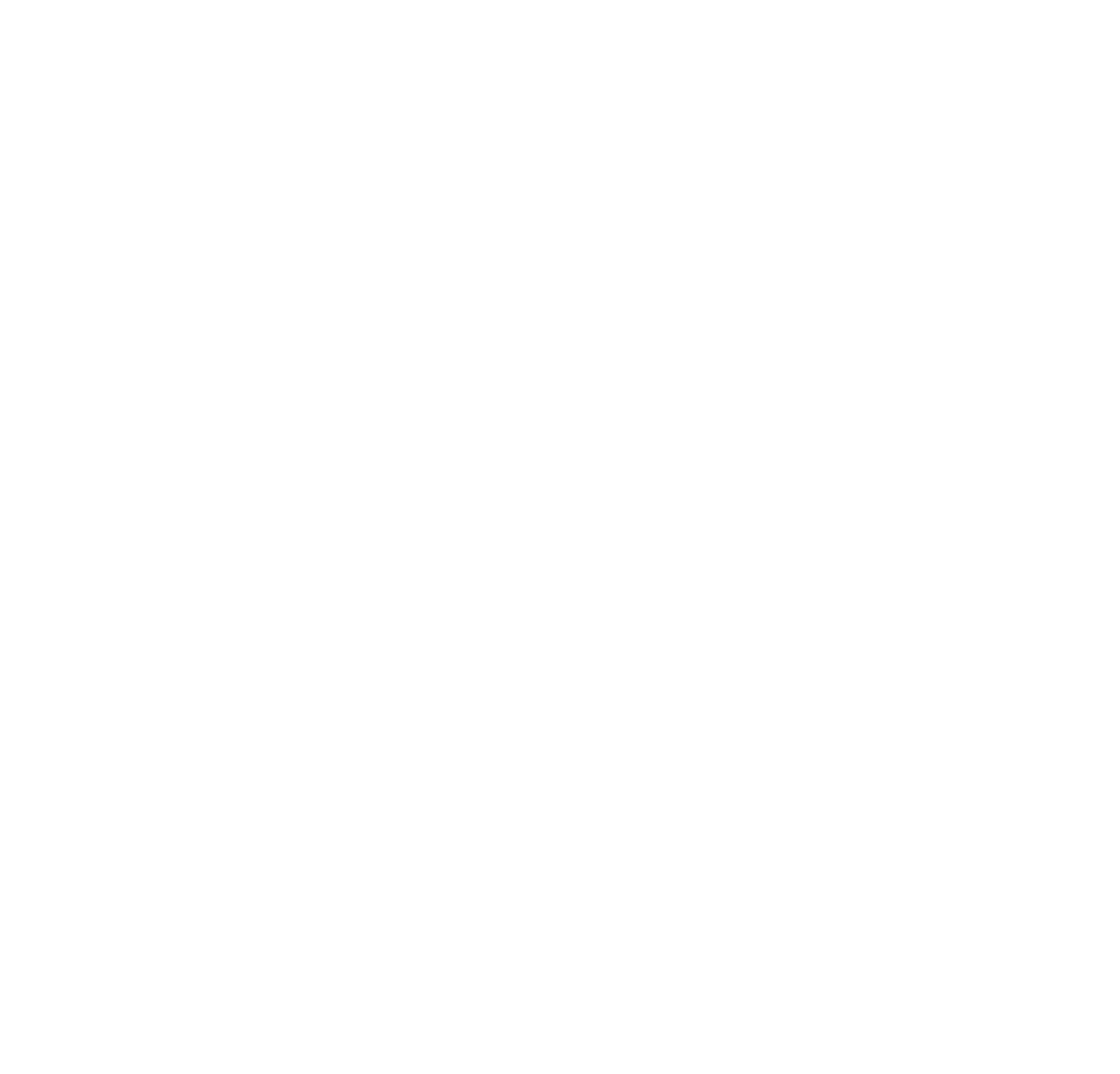

